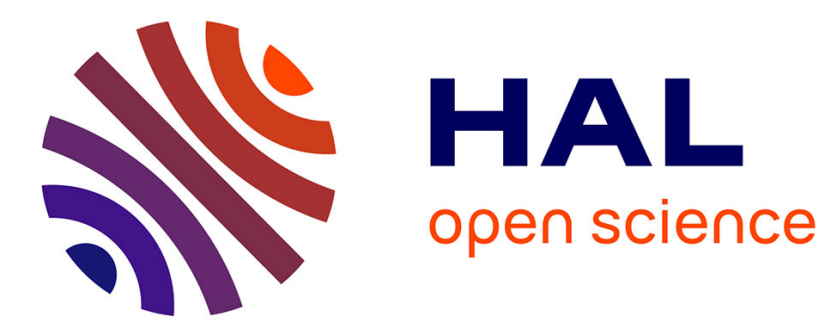

\title{
Physics of the long range proximity effect
}

I. Devyatov, M. Kupriyanov

\section{To cite this version:}

I. Devyatov, M. Kupriyanov. Physics of the long range proximity effect. Journal de Physique IV Proceedings, 1994, 04 (C6), pp.C6-229-C6-234. 10.1051/jp4:1994637 . jpa-00253132

\section{HAL Id: jpa-00253132 https://hal.science/jpa-00253132}

Submitted on 1 Jan 1994

HAL is a multi-disciplinary open access archive for the deposit and dissemination of scientific research documents, whether they are published or not. The documents may come from teaching and research institutions in France or abroad, or from public or private research centers.
L'archive ouverte pluridisciplinaire HAL, est destinée au dépôt et à la diffusion de documents scientifiques de niveau recherche, publiés ou non, émanant des établissements d'enseignement et de recherche français ou étrangers, des laboratoires publics ou privés. 


\title{
Physics of the long range proximity effect
}

\author{
I.A. Devyatov and M. Yu. Kupriyanov
}

Division of Microelectronics, Institute of Nuclear Physics, Moscow State University, Moscow, GSP 119899, Russia

\begin{abstract}
The theoretical model for explanation of the so-called "long range proximity effect" (temperature independent and extremely large supercurrent decay length $\xi_{n}^{*} \cong 10-50 \mathrm{~nm}$ ) of the HTS Josephson junctions with semiconductor oxide interlayer is proposed. It is suggested that the resonant tunneling via a large number of single localized states (LS) in the interlayer is responsible for this effect. We assume that LS are distributed uniformly in the vicinity of Fermy energy and over the interlayer and that their density is small enough for the interaction of electrons occupying different LS be negligible. The interaction of electrons at one LS is also not taken into account. We assume also that the potential barrier in the interlayer has the rectangular form with the height $(V-\mu)$ and the thickness $d$ satisfies the inequalities: $(V-\mu) \ll \mu, d T_{c} /(V-\mu) \leq \alpha \leq d$, where $\mu$ is the chemical potential, $m$ is the electron effective mass, $\alpha^{-1}=(2 m(V-\mu))^{-1 / 2}$ is the effective radius of LS. The proposed model provides the explanation for whole scope of the data concerning "long range proximity effect". We believe that the model can be also applicable for interpretation of the properties of HTS single grain boundary junctions and Low- $T_{c}$ structures with the gapless semiconductor interlayer.
\end{abstract}

\section{INTRODUCTION}

The typical experimental value of the specific single grain boundary resistance $\left(10^{-8}\right.$ $\left.\Omega \mathrm{cm}^{2}\right)$ and the one of SN or SSm interfaces $\left(10^{-8} \Omega \mathrm{cm}^{2}-10^{-10} \Omega \mathrm{cm}^{2}\right)$ are several orders of magnitude higher than the typical values of the $\rho_{n} \xi_{n}^{*}$ product $\left(10^{-10} \Omega \mathrm{cm}^{2}-10^{-12}\right.$ $\Omega \mathrm{cm}^{2}$ ) for the normal metals. So to get the HTS Josephson junctions with the normal junction resistance controlled by the properties of the interlayer one have to use the semiconductor oxides as the weak link material and the so-called shallow-step geometry of the junction to reduce the boundary resistance. The result of intensive study [1-7] of this type structures was the experimental observation of the "long-range proximity effect". This is relatively large $(10-50 \mathrm{~nm})$ and temperature independent supercurrent decay length $\xi_{n}^{*}$ in the interlayers. It was also found that this effect can be observed only in range of the composition of the $\mathrm{Sm}$ materials where the transformation from the metallic to semiconductor type conductivity takes place [6]. 
The last experimental results obtained in $\mathrm{YBa}_{2} \mathrm{Cu}_{3} \mathrm{O}_{7-\delta} / \mathrm{Y}_{0.3} \mathrm{Pr}_{0.7} \mathrm{Ba}_{2} \mathrm{Cu}_{3} \mathrm{O}_{7-\delta} / \mathrm{YBa}_{2} \mathrm{Cu}_{3} \mathrm{O}_{7-\delta}$ shallow-step edge junctions [7] have shown that it would be really possible to fabricate the structures with the semiconductor-type temperature dependence of the normal junction resistance. This evidently indicate that the processes in the junctions are controlled by the properties of the interlayer.

There are several mechanisms of conductivity leading to semiconductor-type temperature dependence of $R_{n}$. They are the activation type conductivity: $R_{n} \propto \exp (\Delta E / k T)$, three- $\left(R_{n} \propto \exp \left(\left(T_{0} / T\right)^{1 / 4}\right)\right)$ or two- $\left(R_{n} \propto \exp \left(\left(T_{0} / T\right)^{1 / 3}\right)\right)$ dimensional hoping-type conductivity and hoping via finite number of the LS . In the last case [8] the conductivity of the junction equal to the sum of the conductivities of the channel $n(\hbar=1)$ :

$R^{-1}=\sum_{n} R_{n}^{-1}, \quad R_{n}^{-1} \propto T^{\left(n-\frac{2}{n+2}\right)} \exp \left(-\frac{2 d}{(n+1) \alpha}\right), \quad \quad \alpha^{-1}=\sqrt{2 m(V-\mu)}$,

where $d$ is the thickness of the I-layer, $m$ is the effective mass of the quasiparticles, $(V-\mu)$ is the barrier height. In particular case $n=2$ from Eq.(1) it follows

$R_{n}^{-1}=A \exp \left(-\frac{2 d}{\alpha}\right)+B \exp \left(-\frac{d}{\alpha}\right)+C T^{4 / 3} \exp \left(-\frac{2 d}{3 \alpha}\right)$.

The temperature independent part of $R_{n}$ is defined by the direct tunneling processes as well as by elastic and inelastictic resonant hoping via one localized center.

The analysis of the experimental dependencies $R_{n}(T)$ within the temperature interval $T \leq 0.5 T_{c}$ for various thicknesses of the interlayer and $R_{n}(d)$ at $T=4.2 K$ have shown that the experimental data [7] fit well by the Eq.(2) with the following values of the constants:
$A \approx 6 \Omega^{-1}$,
$B \approx 10 \Omega^{-1}$,
$C \approx 0.18 \Omega^{-1} K^{-4 / 3}$,
$\alpha \approx 18 n m$.

For $d=75 \mathrm{~nm}$ and $T=4.2 \mathrm{~K}$ these constants provide to the channel resistance of the direct tunneling $R_{0}$, resonant tunneling via one $R_{1}$ and two centers $R_{2}$ the following values:

$R_{0} \approx 690 \Omega, \quad R_{1} \approx 6.5 \Omega, \quad R_{2} \approx 13 \Omega$.

Supposing that the potential well of the barrier has the rectangular form with it's height $(V-\mu)$; from Eq.(1) it is easy to have $(V-\mu)=1 / 2 m \alpha^{2} \approx 10 \mathrm{meV} \approx 100 \mathrm{~K}$. It is obvious that for this rather small values of the barrier height $(V-\mu)$ the type of the interlayer conductivity has to change at temperatures $T \approx 0.5 T_{c} \approx 50 \mathrm{~K}$ from semiconductor to the metal one due to the opening the channels for thermoactivated conductivity . It was observed experimentally [7].

The analysis of the thickness dependence of the critical current gives the usual exponential dependence:

$I_{c} \propto \exp \left(-d / \xi_{n}^{*}\right), \quad \xi_{n}^{*} \approx \alpha \approx 20 \mathrm{~nm}$.

The coincidence of the constants $\alpha$ and $\xi_{n}^{*}$ permits to conclude that it is resonant tunneling which is responsible both for normal and supercurrent transfer via the barrier. From this point of view the "long-range proximity effect" junctions are the SIS structures with a large number of LS in the interlayer and small barrier height. So the experimentally observed in this structures large decay length is simply the effective radius of the LS. The relatively large radius of LS (5) allows to conclude that the Coulomb repulsion of the electrons on the LS characterized by the effective interaction energy $U$ does not completely close this channel for the supercurrent as it follows in the case $U \gg T_{c}$ from the theoretical model [9]. Moreover 
there is the experimental evidence [10] that due to electron-phonon interactions the value of the characteristic energy $U$ can be not only the effectively reduced but even change sign and becomes attractive.

\section{THEORETICAL MODEL}

In proving this assertion we assumed that LS were distributed uniformly in energy (in the vicinity of the Fermy-energy) and over the interlayer and that their density were small enough that the interaction of electrons occupying at different LS is negligible. The interaction of the electrons at one LS is not also taken into account. We assume also that the potential barrier for the quasiparticales in the interlayer has the rectangular form with the height $(V-\mu)$ and the thickness $d$ satisfies the inequalities:

$$
(V-\mu)<<, \quad d T_{c} /(V-\mu) \leq \alpha \leq d,
$$

The last restriction practically means that the dominant channel for the current transport through the junction is the resonant tunneling via one LS on a trajectory so that the processes of the direct tunneling are unimportant.

For calculation of critical current $J_{s}$ under these restrictions it is convenient to use the well-known expression for $J_{s}[11]$ :

$$
\begin{aligned}
J_{c}=-\frac{i e T}{(2 \pi)^{4}} \sum_{\omega} \int d z_{1} d z_{2} d^{2} p_{1} d^{2} p_{2} \Delta\left(z_{1}\right) \Delta^{*}\left(z_{2}\right) * \\
G_{\omega}^{n}\left(p_{1}, p_{2}, z_{1}, z_{2}\right) G_{-\omega}\left(p_{1}, p_{2}, z_{1}, z_{2}\right)\left[\operatorname{sign}\left(z_{1}\right)-\operatorname{sigh}\left(z_{2}\right)\right]
\end{aligned}
$$

Here $p_{1}$ is the transverse momentum, $z_{1}, z_{2}$ are the coordinates reckoned from the middle of the interlayer in the direction perpendicular to the boundaries, $\Delta\left(z_{1}\right)=\Delta \exp (i \varphi / 2)$ and $\Delta\left(z_{2}\right)=\Delta \exp (-i \varphi / 2)$ are the order parameters of the electrodes; $\omega=\pi T(2 n+1)$ are Matsubara frequencies; $G_{\omega}^{n}$ and $G_{\omega}$ are the Furie components of the normal and superconducting Green's functions, taking into account the presence of the LS in the interlayer. These components are related to the imperturbable (in the absence of LS) Green's functions by equations [12]:

$$
\begin{gathered}
G_{\omega}\left(p, p^{\prime}, z, z^{\prime}\right)=(2 \pi)^{2} \delta\left(p-p^{\prime}\right) G_{\omega}^{0}\left(p, z, z^{\prime}\right)+L_{\omega} e^{-i\left(p-p^{\prime}\right) \rho_{0}} G_{\omega}^{0}\left(p, z, z_{0}\right) G_{\omega}^{0}\left(p^{\prime}, z_{0}, z^{\prime}\right), \\
L_{\omega}=\left(\int d^{3} r V(r)\right)\left\{1-\int d^{3} r V(r) G_{w}^{0}\left(r_{0}, r\right)\right\}^{-1} .
\end{gathered}
$$

Here $r_{0}$ is the coordinate of the LS; $\mathrm{V}(\mathrm{r})$ is its local potential; $G_{\omega}^{0}\left(r_{0}, r\right)$ is the imperturbable Green's function in coordinate representation.

The first term in (8) describes the processes of the direct tunneling through the barrier. The value of the supercurrent (7) obtained from this term is exponentially small compared with the term responsible for the resonant tunneling via LS. Ignoring it and taking into account that without loss of generality one can put in (8) $\rho_{0}=0$, it is easy to get:

$$
G_{\omega}\left(p, p^{\prime}, z, z^{\prime}\right)=L_{\omega} G_{\omega}^{0}\left(p, z, z_{0}\right) G_{\omega}^{0}\left(p^{\prime}, z_{0}, z^{\prime}\right) .
$$

Carrying out the calculations following that ones described in the supplement of [13], using the well-known expressions for nondisturbed Green's functions calculated in [14] for 
the rectangular barrier and taking into account the limitations (6) for the amplitude of the resonant scattering $L_{\omega}$ one has:

$$
\begin{aligned}
& L_{\omega}=\frac{2 \pi}{m}\left\{\frac{1}{\alpha_{0}}-\frac{1}{\alpha}-f\left(z_{0}\right)+\left[\frac{\omega}{2 \alpha(V-\mu)}+\frac{2 \omega}{\sqrt{\omega^{2}+\Delta^{2}}} \sqrt{\frac{V-\mu}{\mu}} f\left(z_{0}\right)\right]\right\}^{-1}, \\
& f(z)=\frac{\exp (-2 d / \alpha)}{2}\left\{\frac{\exp (2 z / \alpha)}{d-z}+\frac{\exp (-2 z / \alpha)}{d+z}\right\}, \quad \alpha_{0}^{-1}=\sqrt{2 m\left(V-E_{0}\right)},
\end{aligned}
$$

where $E_{0}$ is the resonance value of the LS energy. The amplitude of the resonant scattering for the NIN structures $L_{\omega}^{n}$ follows from (10) under $\Delta=0$.

The function $f\left(z_{0}\right)$ in the real part (10) is responsible for the renormalization of the energy level of LS which is not important due to the averaging over energy on a final step of the supercurrent calculations. The imaginary part in (10) describes the decay of the resonant state due to the thermal excitations (first term) and tunneling into the electrodes (second term).

The substitution of the equations (9),(10) into the expression of the supercurrent (7) leads to the sinusoidal $J(\varphi)$ relationship with the critical current

$$
\begin{aligned}
J_{c}=-\frac{4 e T \Delta^{2}}{(2 \pi)^{4}} \sum_{\omega} L_{\omega}^{n} L_{\omega} & \left\{\int d^{2} p_{1} \int_{d / 2}^{\infty} G_{\omega}^{n}\left(p_{1}, z_{1}, z_{0}\right) G_{-\omega}\left(p_{1}, z_{1}, z_{0}\right) d z_{1}\right\} * \\
& \left\{\int d^{2} p_{2} \int_{-\infty}^{-d / 2} G_{\omega}^{n}\left(p_{2}, z_{0}, z_{2}\right) G_{-\omega}\left(p_{2}, z_{0}, z_{2}\right) d z_{2}\right\}
\end{aligned}
$$

To calculate $J_{c}$ it is necessary to average (11) over energy and space distributions of LS. The first of these operations gives

$$
\left\langle L_{\infty}^{n} L_{-\infty}\right\rangle_{E_{0}}=\frac{(2 \pi)^{2}}{\alpha m^{3}} n\left(E_{0}\right)\left\{\frac{\omega}{\alpha(V-\mu)}+2 f\left(z_{0}\right) \sqrt{\frac{V-\mu}{\mu}}\left(\frac{\omega}{\sqrt{\left(\omega^{2}+\Delta^{2}\right.}}+\operatorname{sign} \omega\right)\right\}^{-1},
$$

where $n\left(E_{0}\right)$ is the LS density of states. After substitution (12) into (11) and averaging over LS coordinates finally one has:

$$
\begin{aligned}
& \left\langle J_{c}\right\rangle=\frac{4 T \Delta^{2}}{e \alpha \rho_{n}} \sum_{\omega>0} \frac{1}{\omega^{2}+\Delta^{2}} \int_{-d / 2}^{d / 2} \frac{d z_{0}}{\Gamma_{L C}\left(\omega / 2 \pi T_{c}\right)+\beta \operatorname{ch}\left(2 z_{0} / \alpha\right)}, \\
& \rho_{n}^{-1}=\frac{2 e^{2} \pi n\left(E_{0}\right) n\left(z_{0}\right)}{m d} \sqrt{\frac{V-\mu}{\mu}} \exp \left\{-\frac{d}{\alpha}\right\}, \\
& \beta=1+\frac{\omega}{\sqrt{\omega^{2}+\Delta^{2}}}, \quad \Gamma_{L C}=\frac{\pi T_{c}}{V-\mu} \sqrt{\frac{\mu}{V-\mu}} \frac{d}{2 \alpha} \exp \left\{\frac{d}{\alpha}\right\} .
\end{aligned}
$$

Here $\rho_{n}$ is the part of the normal junction resistance defined by the tunneling mechanisms of the decay of the states at LS, $n\left(z_{0}\right)$ is the LS space concentration. 
From (13) it follows that $J_{c}(T)$ depends on dimensionless parameter $\Gamma_{L C}$ proportional to ratio of the escape rates of the electron from LS due to thermal activation and due to resonant tunneling of the quasiparticles from LS into the electrodes.

For the small values of the suppression parameter $\Gamma_{L C}<1$ the last process dominates and the expression for the critical current (13) is essentially simplified:

$$
\left\langle J_{c}\right\rangle=\frac{4 \pi T \Delta^{2}}{e \rho_{n}} \sum_{\omega>0} \frac{1}{\sqrt{\omega^{2}+\Delta^{2}}\left(\omega+\sqrt{\omega^{2}+\Delta^{2}}\right)} .
$$

The temperature dependence of $J_{c} \rho_{n}$ product following from (14) does not differ considerably from the predictions of Ambegaokar - Baratoff (AB) theory [15] (see Fig.1).

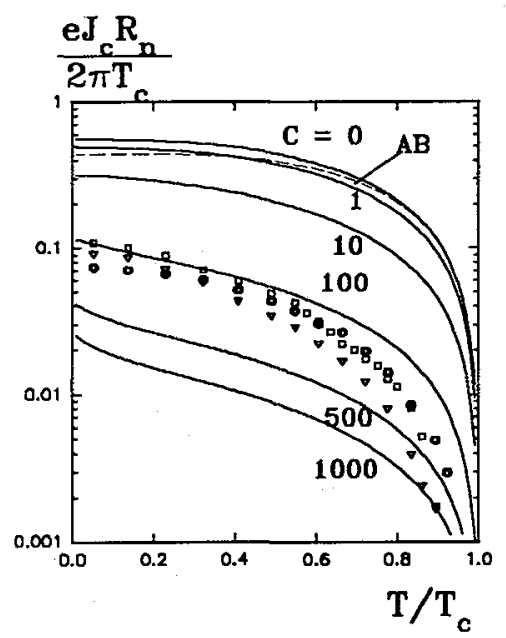

Fig.1. Temperature dependencies of $V_{c}=J_{c} \rho_{n}$ under various values of the suppression parameter $\Gamma_{L C}$.

The normal junction resistance in this limit is defined only by the tunneling processes. The channels for normal and supercurrent transfer coincide with each other, and at $T \approx T_{c}$ the results of the Aslamosov-Larkin theory are followed from (14). At small temperatures $T<<T_{c}$ passing (14) from the summation to the integration over $\omega$ we get:

$$
\left\langle J_{c}(0)\right\rangle=\frac{2 \Delta(0)}{e \rho_{n}}=\frac{4}{\pi} J_{c}^{A B}(0),
$$

where $J_{c}^{A B}(0)$ is the value of the critical current of the SIS junctions that follows from $A B$ theory at $T=0$.

Increase of the parameter $\Gamma_{L C}$ leads to suppression of the resonant tunneling processes due to thermal excitations and reduction $J_{c}$ and in the limit $\Gamma_{L C} \gg \exp (d / \alpha)$, when

$$
T_{c} \gg(V-\mu) \frac{\alpha}{d} \sqrt{\frac{V-\mu}{\mu}},
$$

it becomes exponentially small: 


$$
\left\langle J_{c}\right\rangle=\frac{32 T \Delta^{2}}{e \rho_{n}}(V-\mu) \sqrt{\frac{V-\mu}{\mu}} \sum_{\omega>0} \frac{1}{\left(\omega^{2}+\Delta^{2}\right) \omega} \exp \left\{-\frac{d}{\alpha}\right\} .
$$

At arbitrary values of $\Gamma_{L C}$ numerically calculated $J_{c}(T) \rho_{n}$ product dependencies are shown on Fig.1. Dashed line is the prediction of the $A B$ theory. Circles, triangles and squares are experimental data obtained in $\mathrm{YBa}_{2} \mathrm{Cu}_{3} \mathrm{O}_{7-\delta} / \mathrm{Pro}_{.7} \mathrm{Y}_{0.3} \mathrm{Ba}_{2} \mathrm{Cu}_{3} \mathrm{O}_{7-\delta} / \mathrm{YBa}_{2} \mathrm{Cu}_{3} \mathrm{O}_{7-\delta}$ step-edge junctions [7] with different interlayer thickness. The theoretical curve for $\Gamma_{L C} \approx 100$ fits well the data. It is important to note that the absolute values of the data do not differ greatly despite the essential difference between the interlayer thickness. This fact can not be explain within the framework of existing SNS junctions models [16] as well as within the theory based on the tunneling via several LS on a trajectory [12]. All of them predict the exponential decay of $J_{c} \rho_{n}$ in the experimentally examined thickness interval. In our case both $J_{c}$ and $\rho_{n}$ exponentially depend on $d$ :

$$
J_{c} \propto \exp \left\{-d / \xi_{n}^{*}\right\}, \quad R_{n}^{-1} \propto \exp \{-d / \alpha\}, \quad \xi_{n}^{*} \approx \alpha \approx 20 \mathrm{~mm},
$$

with practically equal characteristic length in the exponent.

It is interesting to point out that in this model it is possible to explain simultaneously the small values of $J_{c} \rho_{n}$ product with its smooth temperature dependencies at $T \leq 0.3 \div 0.4 T_{c}$ and scaling low $J_{c} \rho_{n} \propto 1 / \rho_{n}$.

So, we can conclude that the proposed model based on resonant tunneling via one LS located in the barrier with the relatively small height is practically unique, that provides the explanation all scope of the data [1-7]. We believe that the model can be also applicable for interpretation of the properties of the HTS single grain boundary junctions and Low $-T_{c}$ structures with the gapless semiconductor interlayer.

The authors are grateful to M.Bode, M.Faley, U.Poppe, O.V. Snigirev for the fruitful discussions.

The work was supported in part by Russian State Programs on High Temperature Superconductivity, on Physics of Solid State Nanostructures and by International Science Foundation.

\section{$\underline{\text { References }}$}

1. Kabasawa U., Tarutani Y., Fukazawa et al., Jpn.J.Appl.Phys. 30 (1991) 1670-1675.

2. Kozono Y., Kasai M., Kanke Y.et al., Physica C. 185-189 (1991) 1919-1920.

3. Tanutani S., Fukazawa T., Kobasawa O. et al. Appl.Phys.Lett. 58 (1991) 2707-2709.

4. Boguslavskij Yu.M. and Gao J. Physica C 194 (1992) 268-276.

5. Gao J., Boguslavskij Yu.M., Klopman B.B.G. et al. J.Appl.Phys. 72 (1992) 575-583.

6. Kasai M., Kanke Y., Ohno et al. J.Appl.Phys. 72 (1992) 5344-5349.

7. Stolsel C., Siegel M., Adrian G.et al., "Transport properties of $\mathrm{YBa}_{2} \mathrm{Cu}_{3} \mathrm{O}_{7-\delta} / \mathrm{Y}_{0.3} \mathrm{Pr}_{0.7} \mathrm{Ba}_{2} \mathrm{Cu}_{3} \mathrm{O}_{7-\delta} / \mathrm{YBa}_{2} \mathrm{Cu}_{3} \mathrm{O}_{7-\delta}$ edge junctions", European Conference on Applied Superconductivity, Gottingen 4-8 October 1993 p. 56.

8. Glazman L.I., Matveev K.A. ,Zh.Eksp.Teor.Fiz. 94 (1988) 332-343.

9. Glazman L.I., Matveev K.A., Pis'ma Zh.Eksp.Teor.Fiz. 49 (1989) 570-573.

10. Watkins G.D., Troxell J.R., Phys.Rev.Lett. 44 (1980) 593-595.

11. Aslamasov L.G. ,Fistul M.V., Zh.Eksp.Teor.Fiz. 81 (1981) 382-396.

12. Aslamasov L.G. ,Fistul M.V., Zh.Eksp.Teor.Fiz. 83 (1982) 1170-1176.

13. Lifshitz I.M., Kirpichenkov V.Ya., Zh.Eksp.Teor.Fiz. 77 (1979) 989- 1016.

14. Knauer H., Richter J., Seidel P., Phys.Stat.Sol., 44 (1977) 303-312.

15. Ambegaokar V., Baratoff A., Phys.Rev.Lett. 10 (1963) 486-489.

16. Kupriyanov M.Yu, Likharev K.K , Sov.Phys. Uspekhi 33. (1990) 340-364. 